\title{
LUT
}

University

\section{Virtual Learning Environment for Control Engineering Education}

Nevaranta Niko, Gräsbeck Krister, Haapala Jari-Pekka, Lindh Tuomo, Peltoniemi Pasi, Niemelä Hanna, Pyrhönen Olli

This is a Final draft version of a publication published by IEEE

in 2018 IEEE 18th International Power Electronics and Motion Control Conference (PEMC)

DOI: $10.1109 /$ EPEPEMC.2018.8521905

Copyright of the original publication: (c) 2018 IEEE

Please cite the publication as follows:

Nevaranta, N., Gräsbeck, K., Haapala, J.-P., Lindh, T., Peltoniemi, P., Niemelä, H., Pyrhönen, O. (2018). Virtual Learning Environment for Control Engineering Education. 2018 IEEE 18th International Power Electronics and Motion Control Conference (PEMC). DOI: 10.1109/ EPEPEMC.2018.8521905 


\title{
Virtual Learning Environment for Control Engineering Education
}

\author{
Niko Nevaranta, Krister Gräsbeck, Jari-Pekka Haapala, Tuomo Lindh, \\ Pasi Peltoniemi, Hanna Niemelä and Olli Pyrhönen \\ Department of Electrical Engineering, \\ Lappeenranta University of Technology \\ Lappeenranta, Finland \\ Email:niko.nevaranta@lut.fi
}

\begin{abstract}
Effective teaching of automation and control engineering technology for undergraduate students is of great importance in contributing to the knowledge and skills needed in the engineering profession. Enriching the teaching with animation-based learning material enhances the quality of teaching, bridges the gap between theory and practice, and promotes students' learning results. This paper discusses educational aspects related to the course material in control engineering and recent improvements in teaching methods at Lappeenranta University of Technology (LUT). The paper presents some of the control engineering problems covered in different courses at LUT and discusses perspectives on teaching and learning through virtual learning environments. Based on the student feedback on one course project work where animationbased learning material is used, the implications of virtual models in the course development are analyzed in brief.
\end{abstract}

Keywords-Control Engineering, Teaching, Virtual Learning Environment, Virtual Models

\section{INTRODUCTION}

At Lappeenranta University of Technology (LUT), the undergraduate teaching in control engineering has been primarily based on compact study material founded on established control engineering theory. Enriching the course material with a virtual learning environment provides a viable solution to enhance the quality of teaching, bridge the gap between theory and practice, and ultimately, promote the students' learning results. Similar findings have been reported in the literature on electrical drives teaching [1], where it has been shown that instead of the traditional theoretical approach, it is advantageous to incorporate illustrative examples such as efficient simulation and animation-based learning material in lectures and exercises [6] This notion is also supported by the recent teaching experience at LUT, according to which the use of animation-based virtual models leads to better learning results and higher interest in the topic among students.

Naturally, the basis of any control engineering problem is mathematical modeling of the plant, that is, derivation of the system model used for analysis, estimation, or control design, to name but a few. In a typical classroom situation, mathematical modeling is demonstrated by theoretical presentation of the plant, and the system dynamics is illustrated by equations, simulations, or frequency responses. It has been found that in teaching of control engineering, virtual examples may be beneficial to help the student to understand the control problem [4], [5] and to support situated learning [2], [3]. Further, the engineering education should respond to the evolving skill requirements of the industry and current research activities. Thus, the focus should be on the development of learning environments [6] to support situated and lifelong learning.

The term 'virtual learning environment' is not new in the field of control engineering education, and thus, several papers can be found in the literature. In [7], a 3-D visualization of a simple DC motor servo application has been introduced by using models based on the virtual reality modeling language (VRML). In [8], again, a virtual learning platform is proposed, in which several applications are illustrated by VRML models in order to obtain an interactive animation of control engineering experiments. The paper provides several examples of the modeled laboratory equipment. Another learning environment has been proposed in [9], where a web-based control engineering learning tool is presented. A similar idea has been introduced in [10], where a web-based application is proposed for remote connection of laboratory equipment.

Animation-based visualization is also relevant from an industrial viewpoint. In many practical applications, it is often necessary to perform detailed simulations of the system. This step is often referred to as virtual prototyping [11] or virtual commissioning [12]. As these tasks have become key elements of modern automation systems [13], it is necessary to provide the students with skills that support the increasing use of virtual environments in the industry. This topic has been addressed in [14], where students have been offered a virtual learning environment combined with hardware in loop (HIL) simulations, which give an opportunity to validate the students' control algorithms efficiently and safely prior to the experiments.

The objective of this paper is to demonstrate how animationbased learning material is achieved by using models based on the virtual reality modeling language (VRML) in the Simulink 


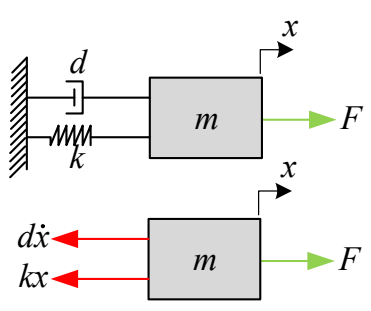

a)

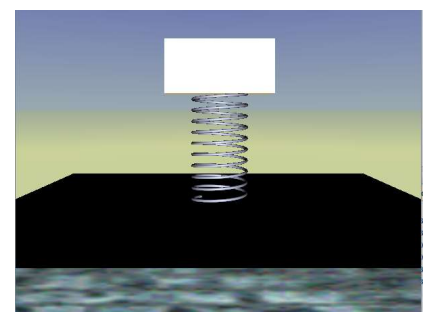

b)
Fig. 1. a) Free-body diagrams of a spring mass-damper system and b) the VRML model of the same dynamics.

environment. The paper introduces some examples of basic control problems, such as the spring-mass-damper and the coupled tank system, which are well-known examples in the control engineering literature. Moreover, some virtual examples of the laboratory equipment used in control engineering courses are given. Finally, a virtual example of a Cartesian robot [15] used as a research test setup is shown.

\section{DERIVATION OF VIRTUAL MODELS}

In the control engineering textbooks [16] there are several established basic control examples to provide an undergraduate student with a solid basis of knowledge and understanding required to proceed to problems that are more complex ones. This section introduces virtual models of a spring-mass-damper system and a coupled tank system, which can be regarded as basic control problems for control engineering students.

\section{A. Spring mass damper system}

The spring-mass-damper system is a well-established control engineering example used to study the dynamics of secondorder systems. Although the dynamics and control of such a system are relatively easy to understand, visualization of the movement gives the students a better indication of how their controller performs. In [17] the derivation of a VRML model of a spring-mass-damper system has been introduced. Based on the guidelines given in the book, a similar virtual model has been built in the Simulink environment. The basis of the dynamics of the virtual model is the second-order differential equation

$$
m \ddot{x}(t)+d \dot{x}(t)+k x(t)=F(t),
$$

where $m$ is the mass, $d$ is the damping constant, $k$ is the spring constant, $x$ is the displacement of the mass, and $F(t)$ is the applied external force. A typical approach to study the dynamics of the system is to use a free-body-diagram of the system as shown in Fig. 1 a), supported by an analysis of transfer functions as well as step and frequency responses. To support the teaching of the system dynamics, a virtual model depicted in Fig. 1 b) has been developed with an interface that gives the students an opportunity to study the behavior with different external forces and system parameters.

In Fig. 2, a simplified interface for the virtual model is shown a) with the corresponding Simulink model b) of the spring-

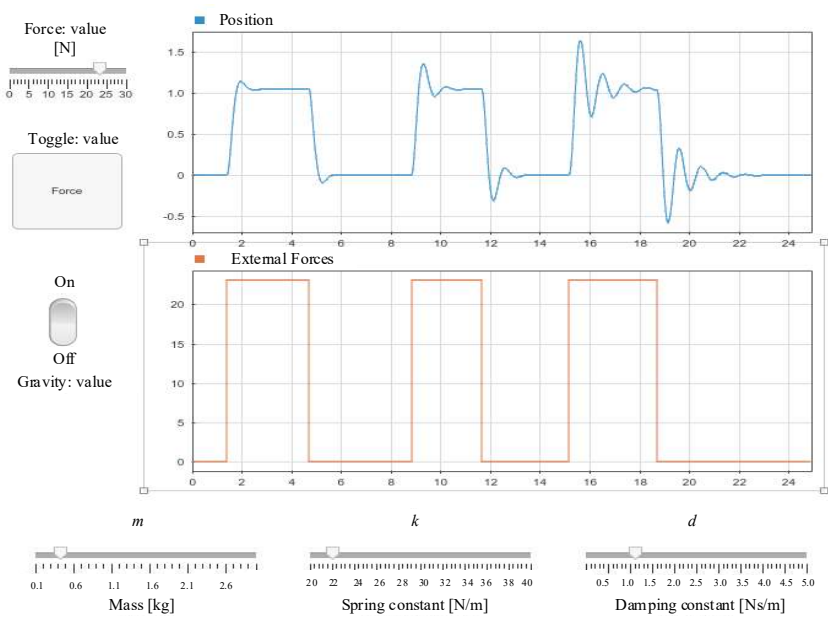

a)

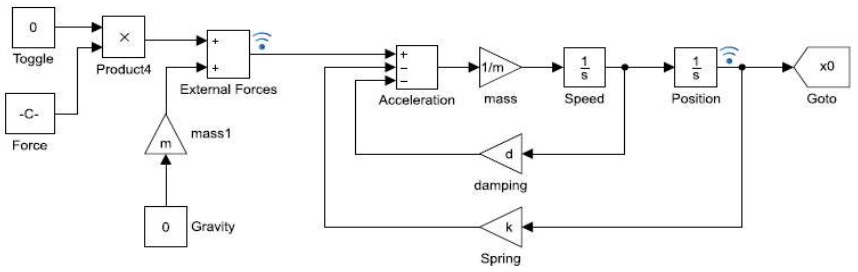

b)

Fig. 2. a) Interface used with the virtual model including a slider selector for the system parameters and the external force. The figures depict the applied forces and the displacement of the mass. b) Simulink model of the dynamics linked to the virtual model shown in Fig. $1 \mathrm{~b}$ ).

mass-damper system. In the example simulations shown in Fig. 2 , the damping constant of the system is varied to demonstrate its impact on the system dynamics. From the learning perspective, the interface is simple and easy to understand.

After the open-loop system dynamics has been studied with the virtual model and frequency responses, the next step is to design a closed-loop control for the system. Typical specifications for the controller are the desired settling time, rise time, overshoot, and steady-state error, which can be derived, for instance, from the second-order system dynamics. The control design is not discussed here, but the virtual interface to study the closed-loop control is shown in Fig. 3. The main interface used for the closed-loop controlled secondorder system is similar to Fig. 2 a), but there is a slider selector

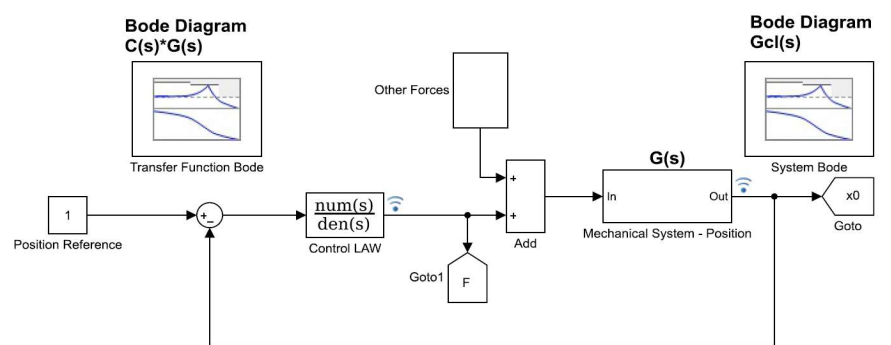

Fig. 3. Closed-loop controlled spring-mass-damper system. The system is linked to the virtual model shown in Fig. 1 b). The Simulink model includes Bode plot functions that can be used to analyze the closed-loop system. 


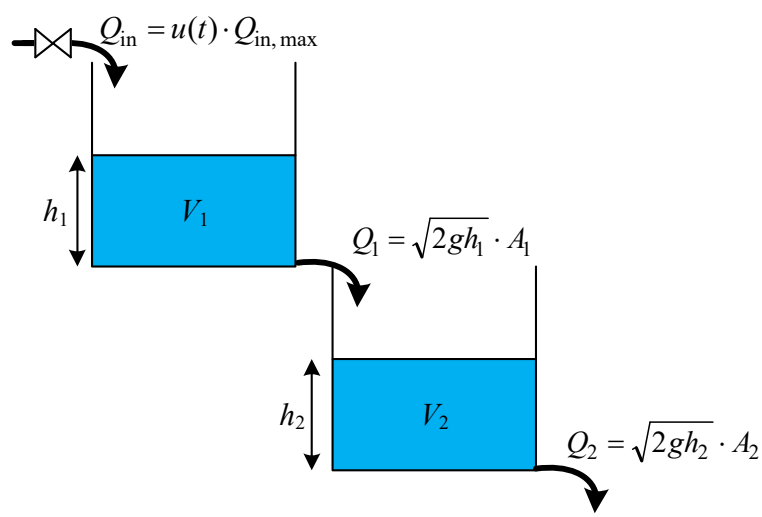

Fig. 4. Principle of a coupled tank system. $Q$ denotes the volumetric flow rate, $V$ the volume of the liquid in the tank, $h$ the height of the liquid level in tank, $g$ the gravitational acceleration, and $A$ the area of the orifice of the tank. $u(\mathrm{t})$ is the position of the valve regulating the input flow rate, and subscripts 1 and 2 are used to denote different tanks.

for the desired position reference, and further, an option to include external forces to introduce disturbances to the system.

The closed-loop Simulink model is similarly linked to the virtual model (see Fig. 1 b), but it includes additional functions to provide more options to analyze the closed-loop system. Thus, Bode diagrams are included in the Simulink model as shown in Fig. 3, which gives the students an opportunity to analyze the system when the controller or system parameters are changed from the interface. This additional function helps the students to understand the behavior of the closed-loop system in the frequency domain.

\section{B. Coupled tank system}

A typical control problem encountered in process industries is the need to control liquid levels in tanks and regulate the flow between tanks. Thus, the modeling and control of the coupled tank system shown in Fig. 4 has become an established control example in textbooks. Depending on the control problem, the tank system may include more valves, but the control objective of the tank system presented in Fig. 4 is to regulate the liquid level in tank 2 by regulating the flow into tank 1 . It is pointed out that the interface includes three valves that can be manually operated to visually study the open-loop dynamics and disturbances under a closed-loop control. This example is used to teach PID-based control, and the student can analyze the designed controller by animation by inserting the control parameters into the interface. The interface has been designed so that the tank parameters can also be changed, providing an option to analyze model-based control laws against plant uncertainties.

\section{VIRTUAL MODELS OF LABORATORY EQUIPMENT}

Because virtual commissioning has become a standard tool in modern automation systems, it is important to consider similar viewpoints in the teaching of control engineering. Naturally, in university-level control engineering education, laboratory

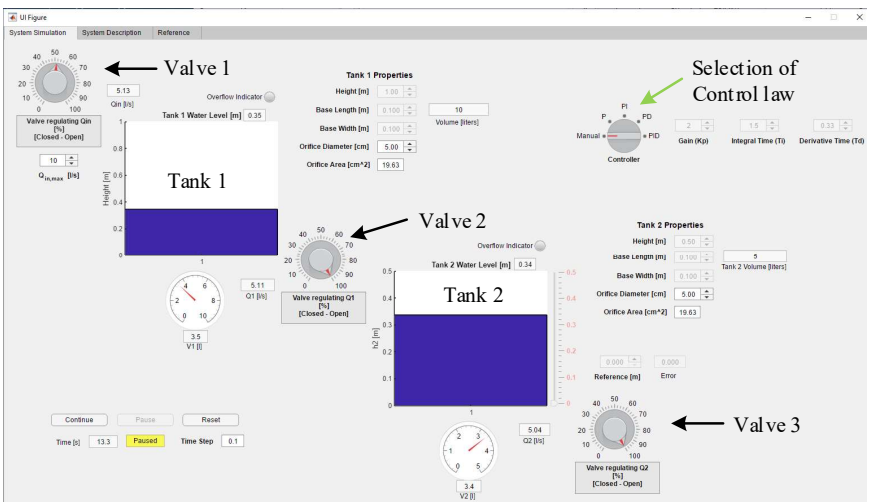

Fig. 5. Graphical interface for the coupled tank problem. The interface has three valves that can be operated manually to study open-loop dynamics or influences of disturbances. The height of the liquid in tank 2 can be regulated by a P-, PI, PD-, or PID-type controller. The interface provides an option to change the properties of the tanks.

experiments play an important role to teach limitations of practical control systems and uncertainties related to mathematical modeling. This section gives some examples of the modeled laboratory equipment used for control engineering education.

\section{A. Twin rotor MIMO system}

A well-known laboratory example in the field of control engineering is the twin rotor MIMO system (TRMS) depicted in Fig. 6 a); it is used to demonstrate the principles of a nonlinear MIMO system with significant cross coupling. In the control engineering community, this application can be regarded as an experimental benchmark problem, and thus, it has been acknowledged in several studies. The TRMS application is used as a project work in the course 'Digital Control Design' at LUT. The VRML model shown in Fig. 6 b) of the TRMS application is used in the lectures as a demonstrative example to illustrate the movement of the application. It has been observed that animation-based visualization of the TRMS application in lectures and exercises promotes the students' motivation for project work.

The students are provided with a virtual model after they have modeled the nonlinear system in Simulink. The use of the VRML model as part of the simulation environment in the controller design and validation steps gives the students visual indication of how their controller performs before it is implemented in the laboratory equipment. The virtual model has been obtained by using SolidWorks, after which it has been transformed into a VRML model in Simulink as shown in Fig. $6 \mathrm{c})$. The model is easy to link to the simulation models derived by the students as it is directly connected to the measured output signals; tail rotor velocity, main rotor velocity, and pitch and yaw angles. The idea for the virtual model connection is adopted from [18]. Further, visualization by Matlab-based 3-D animations has been applied to a somewhat similar case, quadrotor systems, in [19] and [20], which report an improvement in students' learning outcomes as a result of using the visualization tool. 


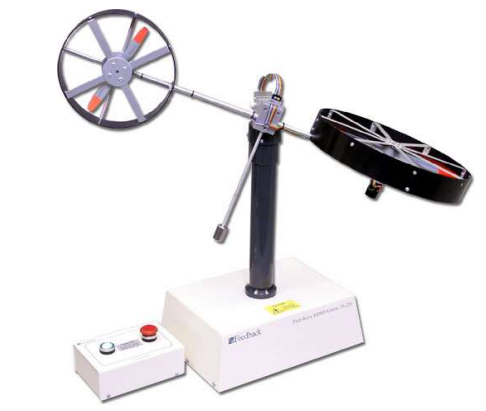

a)

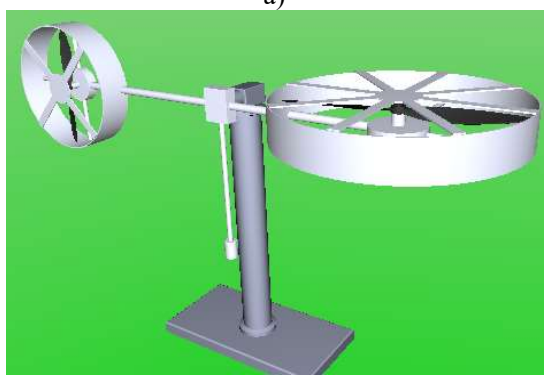

b)

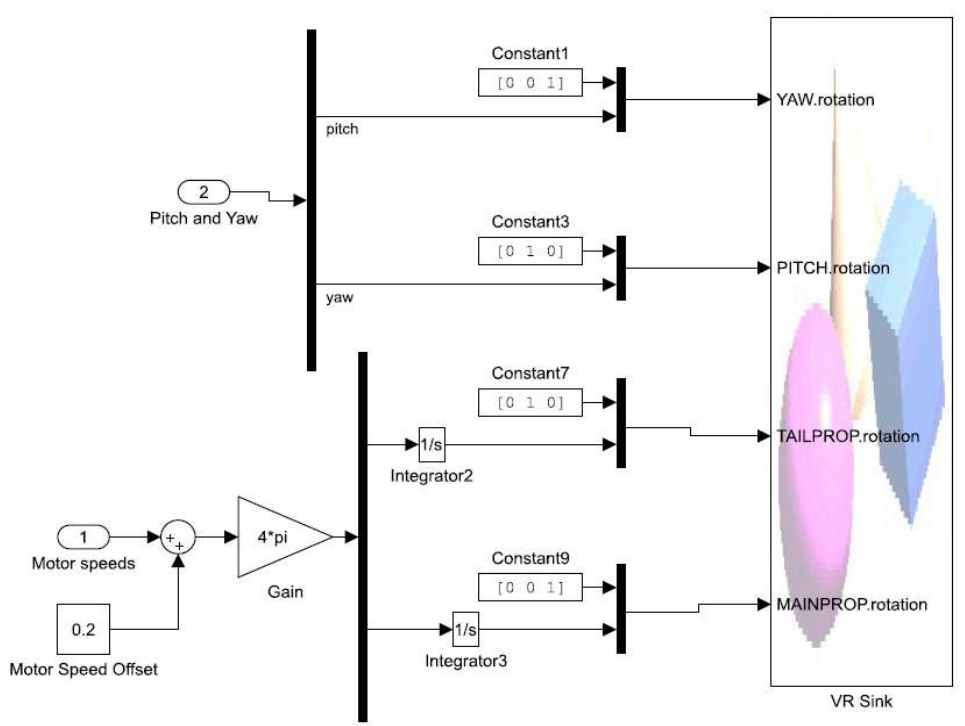

c)

Fig. 6. a) TRMS laboratory equipment manufactured by Feedback Instruments. b) Virtual model of the TMRS application designed in SolidWorks and transformed into a VRML model. c) Example of connecting the Simulink model to the virtual world object.

\section{B. Lego Mindstrom self balancing robot}

An alternative to the TMRS application, the two wheeled selfbalancing Lego Mindstorms robot has been used as a project work in the course 'Digital Control Design'. During the exercises when the controller is validated with an actual robot, it has been found that much time is spent to lift the robot up between the balancing tests. Thus, the $3 \mathrm{D}$-animation, that is, the virtual model, is again useful for virtual commissioning. A virtual model has been derived to help the students to validate the designed control law prior to the experiments with the actual robot and to enhance understanding of the possible errors in the derived control law. Lego robots have been a topic of interest for control engineering students for some time already, and therefore, several virtual model examples are provided, for

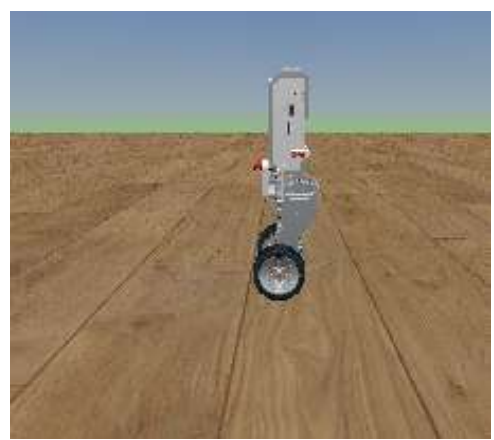

Fig. 7. Virtual model of the Lego Mindstorms self-balancing robot. The model is linked to the Simulink model in a similar way to the TRMS application shown in Fig. 6 c). example, by MathWorks. The model derived for teaching is based on [21] and shown in Fig. 7.

\section{VIRTUAL MOdELS OF THE RESEARCh TEST SETUPS}

Supplementing the learning material with findings from recently completed and ongoing research projects is of great importance in order to enhance the quality of teaching. Moreover, the students can be more interested in the topic when a practical application is considered as a motivating example. In this section, experimental test setups mainly used for research purposes are demonstrated, and the derived virtual model is illustrated.

\section{A. 2-DOF Active magnetic bearing system}

High-speed technology has been a research topic of interest for many years at LUT. In order to teach basic concepts related to the control laws needed for magnetic levitation, a simple twodegree-of-freedom (2-DOF) system is applied. A simple test rig is illustrated in Fig. 8 a). The idea of the setup is to teach modelbased control design and study control issues related to magnetic levitation. To support learning, a virtual model has been developed to visualize levitation, rotor lift-up, and disturbances. The virtual model with a linear controller and a nonlinear model system model is shown Fig. 8 b).

MathWorks also provides a virtual magnetic levitation model based on Humusoft educational laboratory devices, which consists of a coil levitating a steel ball in a magnetic field. This model also offers good visualization of the ball position. However, it is preferable to use a 2-DOF system in the teaching of AMB control systems, as there are two controllable position axes. Moreover, the developed model-based control can be 


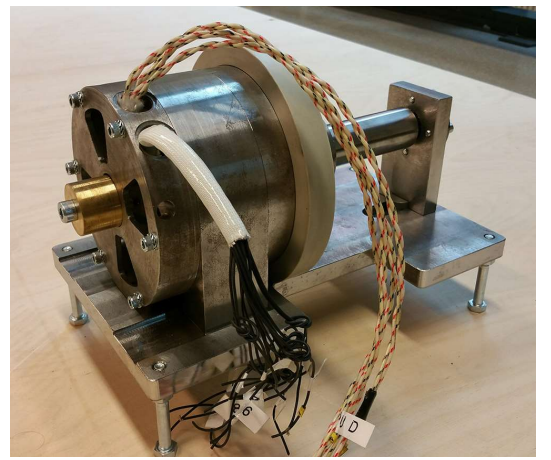

a)

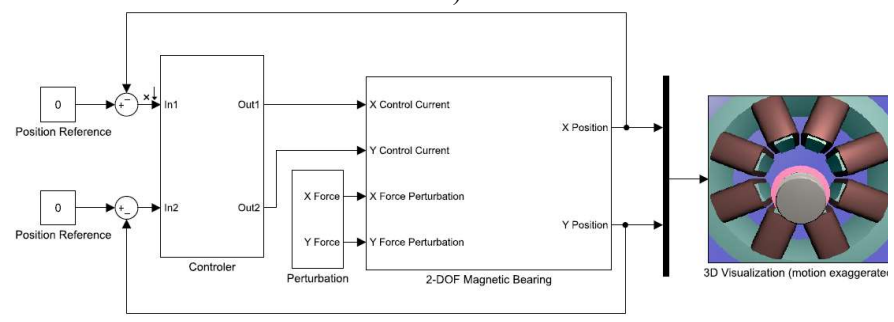

b)

Fig. 8. a) 2-DOF active magnetic bearing system. The rotor is fixed on the other end (right) and the AMB supports the other end (left). b) Virtual model included in the simulation model.

straightforwardly extended to a 4-DOF control system needed for radial direction control in high-speed machines.

\section{B. Cartesian robot application}

Teaching of motion control is also an important element of control engineering instruction at LUT. The test setups used in project assignments are typically linear movement ones, for instance, linear tooth belt drives. The three-axis tooth belt drive Cartesian robot shown in Fig. 9 a) has mainly been used for research purposes [15], but now the application has been incorporated in motion control instruction. Thus, the application has been modeled with SolidWorks and imported into the virtual model depicted in Fig. 9 b). As the tooth belt drive has position-dependent dynamics, the dynamics of the belt has been modeled by a Simulink model using the positiondependent model presented in [22]. These models are linked to the belt pulley dynamics of the SolidWorks model. More information on the components of the experimental system can be found in [15] with a minor modification: the PLC is TwinCat 3.0, which supports the Matlab/Simulink-based programming.

\section{EvaluATION AND FINDINGS}

First, it should be mentioned that most of the presented virtual examples have not yet been incorporated in courses, and thus, there are no student comments available to evaluate the implications of the proposed development. Most of the presented virtual models are to be included in the course 'Digital Control Design' in the academic year of 2018-2019. Nevertheless, the TMRS application discussed above was indeed used in the project work in the academic year of 2017-2018, and these data can be used to evaluate the virtual

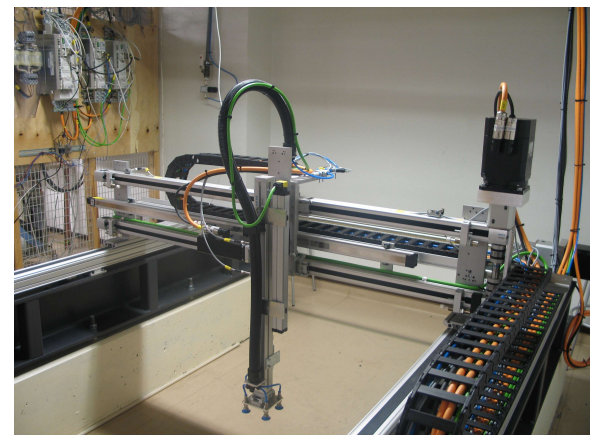

a)

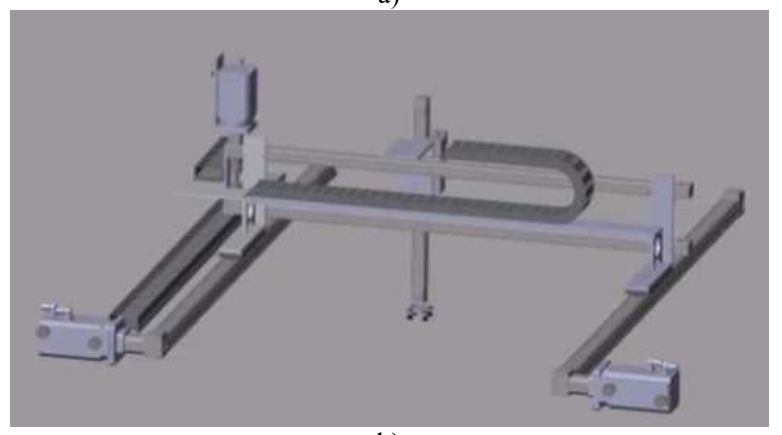

b)

Fig. 9. a) Experimental linear tooth belt Cartesian robot application. b) Virtual model of the system.

model approach from the student perspective. In Table I, a sample of student comments are given on the usefulness of the animation-based visualization of the control problem.

TABLE I

SAMPLE OF STUDENT COMMENTS

Questions:

- In your view, did the animation-based example help you to achieve the learning outcomes?

- What factors affected your level of motivation?

- $\quad$ Should there be more animation-based examples in the course? Comments:

- I am from the Department of Mechanical Engineering, and visualization of the mechanics with the virtual model raised my motivation for the project work and the course. The control problem was easier to understand.

- In my opinion, using an animation-based example is a great idea, and it helped me a lot in my studies.

- I think that there should be more animation-based examples, because sometimes it is hard to understand the theory without visualization.

- The virtual model was especially useful in evaluating the performance of the derived controller.

- $\quad$ There could be more virtual examples of dynamical systems, which could be used for instance to demonstrate oscillation. This could raise the motivation for the topic.

- The virtual model of the TRMS should be used more often by the lecturer in the lectures to support the theory.

- It was useful, but I prefer using signals to analyze the step responses of the derived controller in Simulink. However, the virtual model should be used more in the lectures.

* Obtained from the student feedback after the course (translated from Finnish). 
The comments seem to indicate that the student satisfaction and their overall interest in the topic is promoted when animation-based learning material is used in control engineering instruction. Naturally, the results given in Table I serve only as initial feedback on the usefulness of a virtual example, and thus, actual learning outcomes can be evaluated later when the presented virtual models are included in the course material. The student survey data will be used and further analyzed in the future research. Nevertheless, the initial results in Table I are promising and clearly indicate the usefulness of visual examples.

\section{CONCLUSION}

This paper discussed the opportunities of using animationbased models to support situated learning in control engineering instruction. The paper provided several virtual models of systems that can be advantageous when control engineering is taught to students at any level, from early stages to advanced studies alike. The paper focused on simple control problems, viz. spring-mass-damper and coupled tank systems, which are typical examples in control engineering textbooks. Further, it was shown that virtual models of laboratory equipment are useful for virtual commissioning purposes. Finally, a couple of research test setups were demonstrated, and their virtual models were devised. The models were developed aiming at a computationally simple and easy-to-adopt solution.

Owing to the limited amount of student feedback and data available, it is not possible to evaluate the implications of the developed virtual models for learning outcomes. Nevertheless, most of these models will be included in the courses to be lectured in the academic year of 2018-2019. However, the student feedback presented in this paper suggest that virtual examples could enhance students' learning outcomes.

\section{REFERENCES}

[1] D. Mehmet, "Teaching electric drives control course: Incorporation of active learning into the classroom," IEEE Trans. Educ., vol. 56, no. 4, pp. 459-569, 2012.

[2] J. Anderson, L. Reder and H. Simon, "Situated Learning and education," Jour. of Eng. Education, vol. 25, no. 4, pp. 5-11, May 1996.

[3] Greeno, J. G. and Engeström, Y. I. (2014). Learning in Activity. In Sawyer, R.K. (ed.) The Cambridge Handbook of the Learning Sciences. Second edition. Cambridge: University Press.

[4] M. J. Callaghan, K. McCusker, J. Lopez Losada, J. G. Harkin and S. Wilson, "Teaching engineering education using virtual worlds and virtual learning environments," Int. Conf. on Advances in Comput. Contr. and Telecomm. Tech., Trivandrum, India, 2009, pp. 295-299.
[5] A. Keyhani, M. Marwali, L. Higuera, G. Athalay and G. Baumgartner, "An integrated virtual learning system for the development of motor drive systems," IEEE Trans. Power Systems, vol. 17, no. 1, pp. 1-6, Feb. 2002.

[6] A. Johri, and B.M Olds, "Situated Engineering Learning: Bridging Engineering Education Research and the Learning Sciences," Journal of Engineering Education, Vol. 100, No. 1, pp. 155-185, Jan. 2011.

[7] N-V. Truong, W. Lee and T-T. Nguyen, "Development of virtual control engineering experiments," $11^{\text {th }}$ Int. Conf. on Control, Automation and Systems, Gyenonggi-do, Korea, 2011, pp. 680-685.

[8] C. Schmid, "VCLAB - The virtual control engineering laboratory", Symp. on System Identification (SYSID), California, USA, June, 2000, pp. 515-520.

[9] J.A. Mendez, C. Lorenzo, L. Acosta, S. Torres and E. Gonzales, "A webbased tool for control engineering teaching," Comp. Appl. in Engineering Education, vol. 14, no. 3, pp. 178-187, 2006.

[10] H. Vargas, J. Sanchez, C.A. Jara, F.A. Candelas, F. Torres and S. Dormido, "A network of automatic control web-based laboratories," IEEE Trans. on learning technologies, vol. 4, no. 3, pp. 197-208, July/Sep. 2002.

[11] L. U. Gökdere and C. W. Brice, "Virtual prototyping of motion control systems," Proc. of the IASTED Int. Conf. on Model. and Sim., Philadelphia, PA, 1999, pp. 1-5.

[12] C. G. Lee and S. C. Park, "Survey on the virtual commissioning of manufacturing systems," Jour. of Comp. Design and Engineering, vol. 1, no. 3, pp. 213-222, 2014.

[13] A. Beghi, F. Marcuzzi, P. Martin, F. Tinazzi and M. Zigliotto, "Virtual prototyping of embedded control software in mechatronic systems: A case study," Mechatronics, vol. 43, pp. 99-111, 2017.

[14] Y. Liang, and G.-P. Liu, "Design of Large Scale Virtual Equipment for Interactive HIL Control System Labs," IEEE Trans. on Learning Technologies, vol. PP, no. PP, pp. 1-14, 2017, doi: 10.1109/TLT.2017.2731772.

[15] J. Parkkinen, M. Jokinen, M. Niemelä, T. Lindh and J. Pyrhönen, "Motion synchronization of two linear tooth belt drives using cross-coupled controller," in Proc. EPE, Lille, France, Sep. 2013, pp. 1-7.

[16] R. Dorf, and R. Bishop, Modern control systems, Prentice Hall, 2016.

[17] N. Khaled, Virtual reality and animation for Matlab ${ }^{\circledR}$ and Simulink ${ }^{\circledR}$ users: Visualizations of dynamics models and control simulations, Springer, pp. 21-34, 2012, doi: 10.1007/978-1-4471-2330-9.

[18] B. S. Cazzolato, "Quanser 2DOF Heli, VRML (Virtual reality model)," School of Mechanical engineering, The University of Adelaide, Australia, 2004.

[19] S. Khan, H. Jaffery, A. Hanif, and M. Asif, "Teaching Tool for a Control Systems Laboratory Using a Quadrotor as a Plant in MATLAB," IEEE Trans. on Education, vol. 60, no. 4, Nov. 2017, pp. 249-256.

[20] E. Besada-Portas, J. A. Lopez-Orozco, J. Aranda, and J. M. de la Cruz, "Virtual and remote practices for leaning control topics with a 3DOF quadrotor," in Proc. of $10^{\text {th }}$ IFAC Symp. on Advances in Control Education, Sheffield, UK, Aug. 2013, pp. 78-83.

[21] Y. Yamamoto, "NXTway-GS Model-based design - Control of selfbalancing two wheeled robot built with LEGO Mindstrom NXT," 2008.

[22] N. Nevaranta, J. Parkkinen, T. Lindh, M. Niemelä, O. Pyrhönen, and J. Pyrhönen, "Online estimation of linear tooth-belt drive system parameters," IEEE Trans. Ind. Electron., vol. 62, no. 11, pp. 7214-7223, Nov. 2015. 